\title{
The African Academy of Sciences Research Management Programme in Africa
}

Allen Muyaama Mukhwana ${ }^{1,}{ }^{*}$, Thomas Kariuki ${ }^{1}$, Simon Kay ${ }^{2}$, Angela J. Silva ${ }^{3}$, John Kirkland ${ }^{4}$

${ }^{1}$ The African Academy of Sciences, Nairobi, Kenya

${ }^{2}$ Wellcome Trust, London, UK

${ }^{3}$ MultiCare Institute for Research and Innovation, USA

${ }^{4}$ National Institute of Economic and Social Research, London, UK

"Corresponding author email:

A.Mukhwana@aasciences.ac.ke

Received date: 5 Apr 2019

Published date: 31 Dec 2019

\section{How to cite:}

Mukhwana, A., Kairuki, T., Kay, Silva, A., \& Kirkland, J. (2019). The African Academy of Sciences Research Management Programme in Africa. Journal of Research Management \& Governance, 2(1), 31-33. Retrieved from https:// ejournal.um.edu.my/index.php/ JRMG/article/view/17405

DOI:

https://doi.org/10.22452/

jrmg.vol2no1.4

\begin{abstract}
The Research Management Programme in Africa (ReMPro Africa) is an initiative of The African Academy of Sciences (The AAS) which aims to fill critical gaps in the African research ecosystem to support a vibrant research culture and leadership at universities and research institutions. The AAS recognizes that the business of doing research is allencompassing and the lack of specialist research management profesionals threatens the integrity and quality of research coming out of African institutions. ReMPro Africa aims to transform research management at the institutional level by addressing the key interconnected strands of leadership, sustainability, standards and individual capacity development.
\end{abstract}

Keywords: research management; ecosystems; capacities; standards

\section{Introduction}

The African Academy of Sciences (The AAS) (https://www.aasciences.ac.ke/) has identified research management as a critical gap in building the Continent's research and development ecosystems. In 2017, The AAS set up the Research Management Programme in Africa (ReMPro Africa). ReMPro Africa aims to transform research management at institutional level by addressing the key interconnected strands of leadership, sustainability, standards and individual capacity development.

The development of research management in Africa has been uneven and has generally focused on a limited range of institutions and specific functions (e.g. financial and grants management). This means that other aspects of the research management spectrum, i.e. research strategy and governance, research integrity and ethics, intellectual property, project management, etc. are not developed. ReMPro Africa recognises that research management is strategic and has administrative functions; therefore, to strengthen capabilities requires insight into both dimensions. This means that while considering partnerships and governance, rethinking competencies and career pathways can clearly 
articulate research agendas which are supported by a research management strategy. To achieve research excellence, research management should be an integral component of the research enterprise.

The AAS recognizes that the leadership and administrative requirements of the research enterprise on the researchers go well beyond the actual conduct of research. These demands, as well as the very substantial obligations of scientific publishing, teaching, supervision and mentorship, are required of researchers in the conduct of their research programmes. Unless professional support is available, these demands threaten to, and in many cases do, overwhelm the quality and quantity of research output. This structure is ineffective and unsustainable.

ReMPro Africa aims to systematically create environments that nurture and leverage research. It is designed to build upon previous initiatives in research management and it was developed through comprehensive consultations with stakeholders across the Continent and the globe in workshops conducted by The AAS in February and June 2018 in Nairobi, Kenya and Edinburgh, UK respectively.

ReMPro Africa is based on five principles to address key systemic issues:

- Collaboration within and between African universities and research institutions. Such collaboration ensures the sharing of best practices and strategies to address systematic needs such as robust financial systems that serve the research environment. It is also essential to ensure that initiatives are leveraged, and systematic solutions are applied both broadly (across institutions) and ported forward for the long term.

- Capacity building using evidence-based benchmarks. To address issues that are common across the sector and encourage institutions to develop structures and systems that will affect change.

- African leadership supported by global expertise. To be effective in and for Africa, it must be a programme conceived and implemented in Africa.

- Building on existing structures and expertise. There has already been substantive investment to strengthen institutional research management by external funders and institutions themselves. ReMPro Africa will build on these, rather than seek to 're-invent the wheel'.

- A long-term approach. In some cases, there has been a 'stop-go' approach to research management, reflecting the changing priorities of institutional leaders, national governments and international funders. ReMPro Africa demands a longer-term approach in order to systematically transform research management on the Continent.

With these five principles in mind, four interdependent variables, which constitute the critical success factors for building effective, sustainable research management at an institutional level were identified:

- Institutional leadership. Awareness and engagement at the leadership level is essential for the continuity necessary to strengthen research management functions across successive generations of institutional leadership and senior academic staff.

- Long term commitment. Resources must be committed for the long term to ensure sustainability. This includes not just financial resources, but the creation and investment in a career track for research managers in universities. Institutions must sustain research management functions with or without external (funder or government) support, aligning funder policies with research management practices. 
- High quality standards. Coordinated, high-quality standards and good practices must be created and communicated to all stakeholders within and outside the research institutions.

- Individual capacity development for research managers. A respected career track must be established and maintained to promote the profession of research management, supported by an infrastructure of networks and training opportunities, to ensure that the field attracts and retains talent.

\section{Research Management Programme in Africa (ReMPro Africa)}

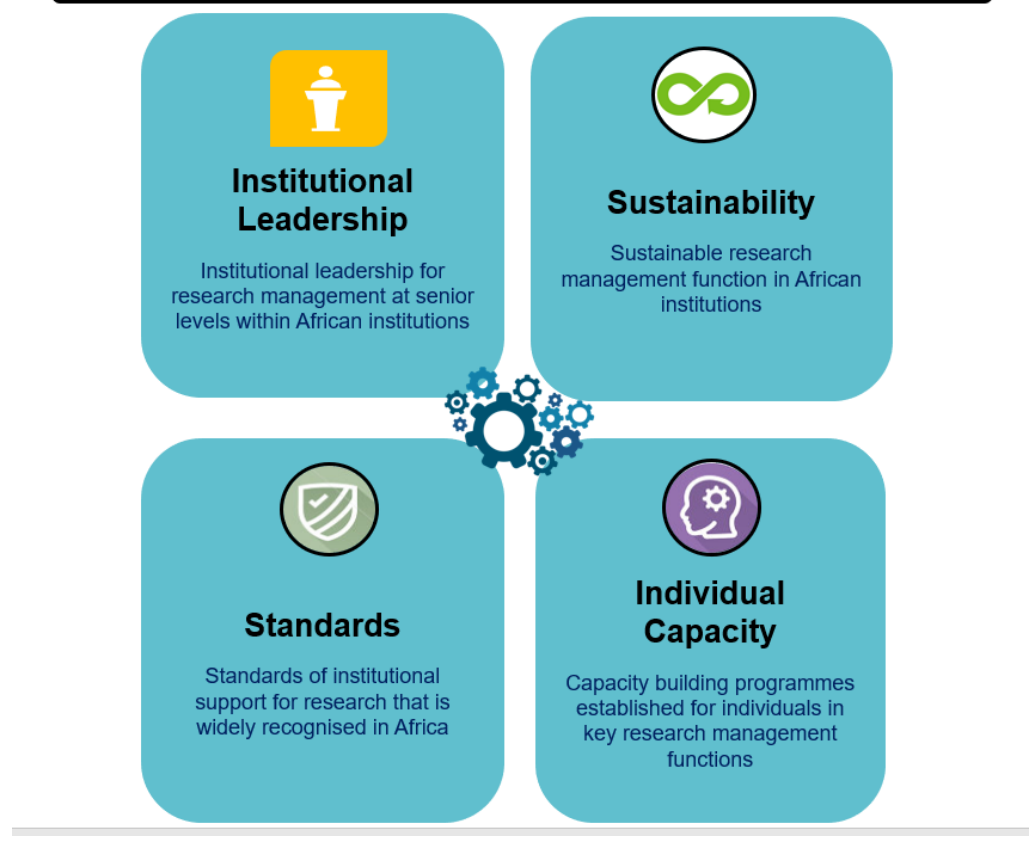

Figure 1: is an illustration of the four interdependent variables.

ReMPro Africa's value addition is in harnessing efforts from several initiatives, and where possible, utilising existing expertise to design interventions and tools that will assist institutions to identify their gaps and work to bridge these gaps. An early outcome will be the Good Research Management Practice Standard (GRMP) that is being developed by the AAS to provide a tool for efficient and effective management of research. This is being designed as a self-assessment tool based on pre-defined criteria (standards) and it allows reflection on the gaps and level of achievement. It is expected to be available for comment during the first half of 2020.

As African institutions increase their research activities; and with increased demand for the production of evidence to inform policy, innovations, etc., it is an opportune time to address the challenges in the research ecosystem. ReMPro Africa is providing a systemic solution to the protracted problem of lack of support for the researchers at African institutions by transforming research management. 